Pensamiento Crítico Vol. 19 № 2, pp. 93-106

\title{
Los determinantes de la tasa de crecimiento de los países de ingresos medios altos y medios bajos en un contexto de crisis internacional: un análisis multivariado
}

The growth rate determinants of the upper and lower middle income countries in the context of the international crisis: A multivariate analysis

Ciro Calero Castillo*

Marco Girio Jesús**

\section{RESUMEN}

En el presente documento se analizan los factores que caracterizan las tasas de crecimiento que alcanzaron los países de ingresos medios altos y medios bajos en el año 2009 mediante la técnica estadística multivariante de análisis discriminante, para lo cual se decidió a priori establecer dos grupos en función de tasas de crecimiento positivas y negativas para poder aplicar esta técnica. Dando como resultado de esta técnica la función discriminante, la cual nos muestran los factores

* Ciro Calero Castillo: Universidad Nacional Mayor de San Marcos.

E-mail: ccaleroc@unmsm.edu.pe, calerociro@yahoo.es.

** Marco Girio Jesús: Universidad Nacional Mayor de San Marcos - Banco Crédito del Perú.

E-mail:mgirio@bcp.com.pe 


\title{
Pensamiento Crítico Vol. 19. $N^{\circ} 2$
}

relevantes para su discriminación, estas son: el gasto público, el índice de justicia, la tasa de fertilidad y el índice de democracia.

Palabras clave: Países de ingresos medios altos y medios bajos, crisis internacional, análisis discriminante.

Clasificación JEL: O4, C380.

\begin{abstract}
In this paper we analyze the factors that characterize the growth rates that reached the upper middle and lower middle income countries in 2009 by the statical technique multivariant the discriminant analysis, for which it was decided a priori to establish two groups based on positive and negative growth rates to apply this technique. Resulting the discriminant function, which show us the relevant factors for discrimination, these are: public spending, the rule of law, the fertility rate and the index of democracy.
\end{abstract}

Keywords: Upper middle and lower middle income countries, international crisis, discriminant analysis.

\section{Introducción}

El año 2009 alrededor del globo todas las economías fueron afectadas por la crisis internacional, unas más que otras, obteniéndose diversos resultados, con países con tasas de crecimiento altas, medias y otras bajas. Muchas de estas son el resultado de políticas macroeconómicas solidas que fueron establecidas durante años atrás que aprovecharon épocas de bonanza y desarrollando ciertos aspectos estructurales de cada una de sus economías y que les permitió ser punto de atracción de capitales extranjeros que permitieron un dinamismo de sus tasas de crecimiento.

Como bien establece Moran Samayoa (2004), la importancia del crecimiento económico radica en su efecto directo sobre el bienestar general. En tanto mayor sea la diferencia entre la tasa de crecimiento de la economía y la tasa de crecimiento de la población, mayor será el incremento en el bienestar general del país. Además, se ha demostrado que existe una relación positiva entre crecimiento económico y reducción de la pobreza. 


\section{Ciro Calero / Marco Girio}

El principal objetivo de cada país es formar un conjunto de fundamentos económicos adecuados, y después de eso, la implementación de medidas económicas apropiadas que sean aceptadas para un país en un determinado momento del tiempo. Aunque no exista un conjunto único de fundamentos económicos en el mundo, tampoco se puede aplicar una solución única en todos los países al mismo tiempo. Aun peor, es más difícil encontrar una solución única en el contexto de una crisis financiera.

Por años el crecimiento económico y sus determinantes han captado la atención de la literatura macroeconómica. Los determinantes del crecimiento son importantes porque permiten caracterizar las economías, inferir acerca de la sostenibilidad del crecimiento y detectar posibles fuentes de crecimiento que aceleren la actividad económica en un país. Por ende, aquí analizaremos que variables caracterizan a los países de ingresos medios altos y medios bajos agrupados en dos, definiéndolos mediante su tasa de crecimiento, mediante una regla fácil de países con crecimientos y sin crecimiento, en donde los primeros son aquellos que tienen una tasa positiva y mayor que cero, mientras que los países sin crecimiento son aquellos que tienen menor que una tasa de $0 \%$, para un periodo de crisis internacional, lo haremos mediante la técnica de análisis discriminante para ver que variables son las que discriminan la variable respuesta. Las variables que se usarán son de la literatura y dentro del marco teórico y los datos fueron obtenidos del Banco Mundial para el periodo del 2009, época en crisis internacional para 105 países.

\section{Fundamentos empíricos}

Muchas teorías del crecimiento económico han sido desarrolladas tanto a nivel teórico como a nivel empírico.

Aquí detallaremos de forma breve el documento en el cual nos hemos basado para escoger las variables independientes para nuestra estimación. Este estudio fue desarrollado por Robert Barro (1999), quien analiza cerca de 100 países con el método de panel data, además hace un análisis puntual para Chile para un espacio temporal de estudio de 1960 a 1995. En este documento sostiene y fundamenta los principales resultados empíricos y toma en cuenta el nivel de gasto de gobierno, la seguridad de los derechos de las personas y el sistema legal al cual llama "the rule of law", el índice de democracia, la tasa de inflación, proxy de la calidad y nivel de la educación, la tasa de fertilidad, la inversión, los términos de intercambio, la tasas de fertilidad, obteniendo los siguientes resultados: 


\title{
Pensamiento Crítico Vol. 19. $N^{\circ} 2$
}

\author{
CUADRO 1
}

Resultados encontrados por Robert Barro

\begin{tabular}{|c|c|c|}
\hline \multicolumn{3}{|c|}{ PANEL REGRESSIONS FOR GROWTH RATE AND INVESTMENT RATIO } \\
\hline Independent variable & $\begin{array}{l}\text { Estimated Coefficient } \\
\text { for Growth Rate }\end{array}$ & $\begin{array}{l}\text { Estimated Coefficient } \\
\text { for Investmeat Ratio }\end{array}$ \\
\hline $\log (p c r$ capita GDP) & $0.124(0.027)$ & $0.188(0.083)$ \\
\hline log(per capita GDP) squared & $-0.0095(0.0018)$ & $=0.0110(0.0053)$ \\
\hline govt. consumption/GDP & $-0.149(0.023)$ & $-0.271(0.072)$ \\
\hline rule-of-law index & $0.0172(0.0053)$ & $0.064(0.020)$ \\
\hline democracy index & $0.054(0.029)$ & $0.072(0.078)$ \\
\hline democracy index squared & $-0.048(0.026)$ & $-0.086(0.068)$ \\
\hline inflation rate & $-0.037(0.010)$ & $-0.058(0.027)$ \\
\hline years of schooling & $0.0072(0.0017)$ & $-0.0013(0.0058)$ \\
\hline $\log$ (total fertility rate) & $-0.0251(0.0047)$ & $-0.0531(0.0140)$ \\
\hline investmen $/ G D P$ & $0.059(0.022)$ & $\ldots$ \\
\hline growth rate of terms of trade & $0.165(0.028)$ & $0.052(0.067)$ \\
\hline numbers of observations & $79,87,84$ & $79,87,85$ \\
\hline $\mathrm{R}^{2}$ & $0.67,0.48,0.42$ & $0.52,0.60,0.65$ \\
\hline
\end{tabular}

Para un mayor detalle de los fundamentos teóricos de las variables, consultar el paper de Barro (1999) "Determinants of economic growth: Implications of the Global Evidence for Chile".

Lo que se hará es realizar una estimación con una técnica diferente para corroborar que los hallazgos por Barro aún se siguen manteniendo, pero aplicados para una muestra en donde participan 105 países de ingresos medios altos y bajos.

\section{Método}

Para identificar los determinantes de la tasa de crecimiento en un contexto de crisis internacional, se utilizará la técnica estadística multivariante de análisis discriminante. A continuación se explican los supuestos de este modelo y sus principales implicaciones.

El análisis discriminante es una técnica multivariante orientada fundamentalmente a lograr dos objetivos básicos:

a. Explicar la influencia de un conjunto de variables cuantitativas sobre una variable cualitativa.

b. Predecir la pertenencia de los sujetos a una de las categorías de la variable criterio a partir de los valores registrados en las variables predictivas. 


\section{Ciro Calero / Marco Girio}

Antes de la utilización de cualquier prueba estadística, se debe comprobar el cumplimiento de los supuestos básicos de aplicación:

(i) Las variables independientes o predictivas deben seguir una distribución normal multivariante.

(ii) Las matrices de covarianzas deben ser iguales en todos los grupos.

Sin embargo, el análisis discriminante es considerado una técnica robusta que no se ve gravemente afectada si alguno de los supuestos anteriores no se cumple. En este caso, como tenemos solo dos grupos, el primer supuesto no es necesario comprobarlo, ya que Fischer demostró que para el caso de dos grupos la violación de este supuesto no lo distorsiona en gran magnitud.

Mientras que para el segundo supuesto es recomendable aplicar el test de $\mathrm{M}$. de Box para comprobar el segundo supuesto. El test de M. de Box parte del supuesto de que las matrices de covarianzas son iguales y se basa en el cálculo de los determinantes de covarianza de cada grupo, el valor obtenido se aproxima a la F de Snedecor.

Geométricamente se busca una buena dirección sobre la que proyectar los datos de los grupos conocidos y de los que queremos clasificar. Se clasifica en función de qué grupo está más cerca de esa dirección.

Una buena dirección tiene que:

- Separar bien las medias.

- Teniendo en cuenta la variabilidad.

El nuevo dato se clasifica dentro del grupo con la media más próxima en la proyección.

Lo que nos daría para el caso de dos grupos, como es en nuestro análisis, una función discriminante, compuesta de las variables independientes que caracterizan a la variable de estudio. 
CUADRO 2

Función lineal de Fischer para dos grupos con matrices de covarianzas iguales

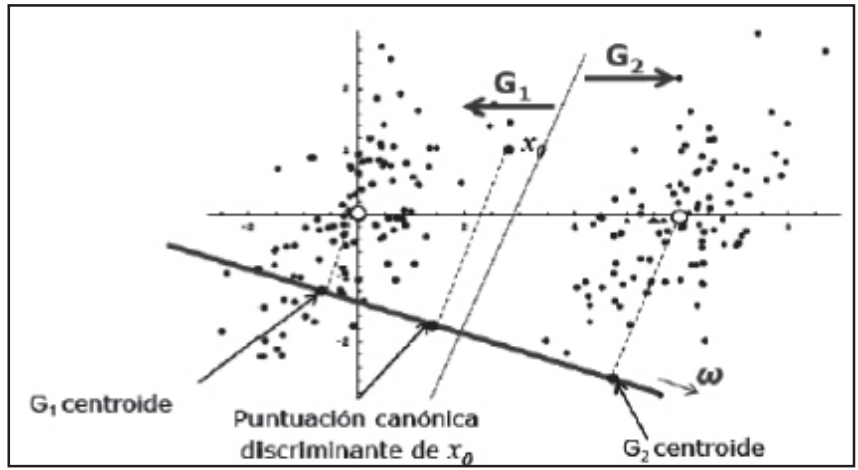

\section{Resultados}

De las múltiples estimaciones que se hicieron se presentan las variables y el mejor modelo estimado. En el primer cuadro se pueden observar los datos obtenidos con respecto a la tasa de crecimiento de cada país, analizado lo que nos brinda un

\section{CUADRO 3}

Tasas de crecimiento de los países analizados. Año 2009

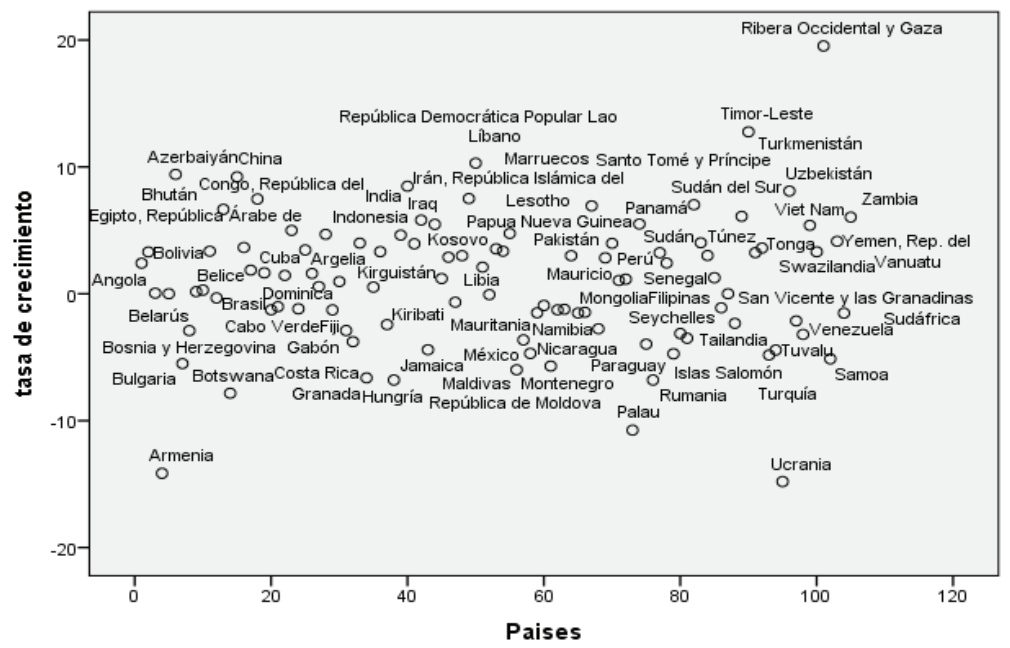




\section{Ciro Calero / Marco Girio}

panorama de su distribución y sus niveles de tasas de crecimiento, observándose tasas de crecimiento negativas y positivas, en donde podemos encontrar a Perú en el grupo de los países que tuvieron una tasa de crecimiento en el 2009.

En el segundo cuadro se puede observar a nivel descriptivo que las variables que caracterizan los dos grupos que hemos definido sí son relevantes, por lo cual a primera vista se podría decir que estos dos grupos si difieren y que estas variables independientes sí las diferencia, esto se puede apreciar en su media y desviación estándar. Este análisis exploratorio nos sirve a priori para establecer y/o confirmar los posibles determinantes para las funciones de discriminación.

\section{CUADRO 4}

Análisis descriptivo de los grupos definidos y variables independientes

\begin{tabular}{|ll|r|r|r|r|}
\multicolumn{9}{|c|}{ Group Statistics } \\
\hline & & & \multicolumn{2}{c|}{ Valid N (listwise) } \\
\cline { 4 - 6 } grupo & & Mean & Std. Deviation & Unweighted & Weighted \\
\hline Pais con crecimiento & terminos de intercambio & 117,9083 & 31,12314 & 33 & 33,000 \\
& inflacion & 5,9252 & 5,60618 & 33 & 33,000 \\
& indice de justicia & 35,4876 & 18,74202 & 33 & 33,000 \\
& gasto publico & 17,8970 & 9,50428 & 33 & 33,000 \\
& tasa de fertilidad & 3,1304 & 1,29450 & 33 & 33,000 \\
& inversion & 26,6770 & 10,22572 & 33 & 33,000 \\
& indice de democracia &, 3587 &, 11062 & 33 & 33,000 \\
\hline Pais sin crecimiento & terminos de intercambio & 101,2755 & 29,50277 & 25 & 25,000 \\
& inflacion & 4,4283 & 3,99197 & 25 & 25,000 \\
& indice de justicia & 46,7678 & 15,94719 & 25 & 25,000 \\
& gasto publico & 28,9125 & 10,54262 & 25 & 25,000 \\
& tasa de fertilidad & 2,0683 &, 60916 & 25 & 25,000 \\
& inversion & 23,5222 & 7,82655 & 25 & 25,000 \\
& indice de democracia &, 4705 &, 13217 & 25 & 25,000 \\
\hline Total & terminos de intercambio & 110,7390 & 31,29426 & 58 & 58,000 \\
& inflacion & 5,2800 & 4,99134 & 58 & 58,000 \\
& indice de justicia & 40,3497 & 18,33119 & 58 & 58,000 \\
& gasto publico & 22,6451 & 11,30448 & 58 & 58,000 \\
& tasa de fertilidad & 2,6726 & 1,17410 & 58 & 58,000 \\
& inversion & 25,3172 & 9,32623 & 58 & 58,000 \\
& indice de democracia &, 4069 &, 13171 & 58 & 58,000 \\
\hline
\end{tabular}




\section{Pensamiento Crítico Vol. 19. No 2}

Empezaremos con la estimación del modelo de análisis discriminante en donde analizaremos primero la prueba para la igualdad de grupos en medias, donde la hipótesis nula del test Lambda de Wilks es que las variables términos de intercambio, inflación, índice de justicia, gasto público, tasa de fertilidad, inversión e índice de democracia son iguales en los dos grupos.

Para las variables gasto público, tasas de fertilidad, índice de democracia como el sig es menor a 0.05 , entonces se rechaza la hipótesis nula, entonces existen diferencias significativas entre los grupos. Mientras que para las otras variables no hay diferencias. Pero no olvidar que estos son a un nivel de análisis univariados y no multivariados.

CUADRO 5

Tests of Equality of Group Means

\begin{tabular}{|l|r|r|r|r|r|}
\hline & \multicolumn{1}{|c|}{$\begin{array}{c}\text { Wilks' } \\
\text { Lambda }\end{array}$} & \multicolumn{1}{c|}{ F } & \multicolumn{1}{c|}{ df1 } & \multicolumn{1}{c|}{ df2 } & \multicolumn{1}{c|}{ Sig. } \\
\hline terminos de intercambio &, 930 & 4,247 & 1 & 56 &, 044 \\
inflacion & 978 & 1,286 & 1 & 56 &, 262 \\
indice de justicia &, 906 & 5,844 & 1 & 56 &, 019 \\
gasto publico &, 763 & 17,390 & 1 & 56 &, 000 \\
tasa de fertilidad &, 796 & 14,371 & 1 & 56 &, 000 \\
inversion &, 971 & 1,646 & 1 & 56 &, 205 \\
indice de democracia &, 820 & 12,284 & 1 & 56 &, 001 \\
\hline
\end{tabular}

Seguiremos con la comprobación de uno de los supuestos para la aplicación de este análisis multivariado que es el de la matriz de covarianzas sean iguales, esto se corroborará mediante el $\mathrm{M}$ de Box, donde la Hipótesis Nula $(\mathrm{Ho})$ plantea que la matriz de covarianzas sean iguales.

Como se observa en el cuadro posterior el sig es mayor a 0.05 , por lo cual se acepta la hipótesis nula, por tanto se concluye que la matriz de covarianzas son iguales. Por ende, cumpliría el supuesto planteado para la estimación mediante esta técnica. Mientras que el primer supuesto de normalidad multivariada no será probado debido a que para un análisis como este, en donde existen dos grupos, Fisher demostró que no afecta la violación de este supuesto. 


\section{Ciro Calero / Marco Girio}

CUADRO 6

Prueba M de Box

Test Results

\begin{tabular}{|ll|r|}
\hline Box's M & & 24,695 \\
F & Approx. & 2,273 \\
& df1 & 10 \\
& df2 & 12588,715 \\
& Sig. &, 012 \\
\hline
\end{tabular}

Tests null hypothesis of equal population covariance matrices.

A continuación estimamos el análisis por pasos para ver qué variables son las que diferencian estos dos grupos y conforman las funciones discriminantes. Donde se puede observar que las variables que nos servirán para discriminar las cuales son el gasto público, la tasa de fertilidad y el índice de democracia y el índice de democracia y en el paso 4 de acuerdo al estadístico $\mathrm{F}$ nos indica que no entra ninguna otra variable para ser significativa.

La tabla Lambda de Wilks muestra el estadístico Lambda de Wilks global para el modelo generado en cada escalón, independientemente de que se haya optado por cualquier estadístico como método de selección de variables. Como se observa, el estadístico Lambda de Wilks va disminuyendo en cada escalón, lo cual es símbolo de que a medida que se va incluyendo variables al modelo, los grupos van estando cada vez menos solapados. En la columna F exacta se encuentra el valor transformado de lambda de Wilks y su significancia. Los valores del estadístico se refieren al estadístico global y no al cambio en el estadístico.

Pasaremos a analizar el autovalor de la función y la correlación canónica. El autovalor de una función se interpreta como la parte de variabilidad total de la nube de puntos proyectada sobre el conjunto de todas las funciones atribuible a la función. Si su valor es grande, la función discriminará mucho. Su valor es el cociente entre la variación debida a las diferencias entre los grupos y la variación que se da dentro de cada grupo combinada en una única cantidad. Este estadístico se diferencia de la F del análisis de varianza multivariante en que no intervienen los grados de libertad. Su interés principal

\section{IOI}


Pensamiento Crítico Vol. 19. № 2

CUADRO 7

Estimación del modelo por pasos

Wilks' Lambda

\begin{tabular}{|c|c|c|c|c|c|c|c|c|c|}
\hline \multirow[b]{2}{*}{ Step } & \multirow{2}{*}{$\begin{array}{c}\text { Number of } \\
\text { Variables }\end{array}$} & \multirow[b]{2}{*}{ Lambda } & \multirow[b]{2}{*}{ df1 } & \multirow[b]{2}{*}{$\mathrm{df} 2$} & \multirow[b]{2}{*}{$\mathrm{df} 3$} & \multicolumn{4}{|c|}{ Exact $\mathrm{F}$} \\
\hline & & & & & & Statistic & df1 & $\mathrm{df} 2$ & Sig. \\
\hline 1 & 1 &, 763 & 1 & 1 & 56 & 17,390 & 1 & 56,000 &, 000 \\
\hline 2 & 2 &, 679 & 2 & 1 & 56 & 13,010 & 2 & 55,000 &, 000 \\
\hline 3 & 3 &, 626 & 3 & 1 & 56 & 10,766 & 3 & 54,000 &, 000 \\
\hline 4 & 4 &, 578 & 4 & 1 & 56 & 9,669 & 4 & 53,000 &, 000 \\
\hline
\end{tabular}

Variables Not in the Analysis

\begin{tabular}{|c|c|c|c|c|c|}
\hline Step & & Tolerance & $\begin{array}{c}\text { Min. } \\
\text { Tolerance }\end{array}$ & F to Enter & $\begin{array}{l}\text { Wilks' } \\
\text { Lambda }\end{array}$ \\
\hline \multirow[t]{7}{*}{0} & terminos de intercambio & 1,000 & 1,000 & 4,247 &, 930 \\
\hline & inflacion & 1,000 & 1,000 & 1,286 & ,978 \\
\hline & indice de justicia & 1,000 & 1,000 & 5,844 & ,906 \\
\hline & gasto publico & 1,000 & 1,000 & 17,390 &, 763 \\
\hline & tasa de fertilidad & 1,000 & 1,000 & 14,371 &, 796 \\
\hline & inversion & 1,000 & 1,000 & 1,646 &, 971 \\
\hline & indice de democracia & 1,000 & 1,000 & 12,284 &, 820 \\
\hline \multirow[t]{6}{*}{1} & terminos de intercambio & ,969 &, 969 & 6,036 &, 688 \\
\hline & inflacion & ,983 &, 983 & 2,139 &, 734 \\
\hline & indice de justicia &, 943 &, 943 & 1,599 &, 741 \\
\hline & tasa de fertilidad & 960 &, 960 & 6,822 &, 679 \\
\hline & inversion & ,998 &, 998 &, 933 &, 750 \\
\hline & indice de democracia &, 975 &, 975 & 6,242 &, 685 \\
\hline \multirow[t]{5}{*}{2} & terminos de intercambio &, 807 &, 799 & 2,021 &, 654 \\
\hline & inflacion &, 783 &, 764 & ,088 &, 678 \\
\hline & indice de justicia &, 876 &, 876 &, 306 &, 675 \\
\hline & inversion &, 932 &, 896 & 2,511 &, 649 \\
\hline & indice de democracia &, 969 &, 942 & 4,583 &, 626 \\
\hline \multirow[t]{4}{*}{3} & terminos de intercambio &, 764 &, 764 &, 823 &, 616 \\
\hline & inflacion &, 780 &, 762 &, 028 &, 625 \\
\hline & indice de justicia &, 272 &, 272 & 4,364 &, 578 \\
\hline & inversion &, 922 &, 894 & 2,994 &, 592 \\
\hline \multirow[t]{3}{*}{4} & terminos de intercambio &, 717 &, 255 &, 147 &, 577 \\
\hline & inflacion &, 771 &, 269 &, 137 &, 577 \\
\hline & inversion & ,870 &, 257 & 1,467 &, 562 \\
\hline
\end{tabular}




\section{Ciro Calero / Marco Girio}

radica en que permite comparar cómo se distribuye la dispersión intergrupos cuando existe más de una función. Aunque un autovalor tiene un mínimo de cero, no tiene un máximo, lo cual lo hace difícilmente interpretable por sí solo. Por esta razón, se acostumbra utilizar el estadístico lambda de Wilks, que se encuentra estrechamente relacionado con los autovalores.

La correlación canónica es la correlación entre la combinación lineal de las variables independientes (la función discriminante) y una combinación lineal de variables indicador (unos y ceros) que recogen la pertenencia de los sujetos s los grupos. En el caso de dos grupos la correlación canónica es la correlación simple entre las puntuaciones discriminantes y una variable con códigos 1 y 0 , según cada caso pertenezca a un grupo o a otro. Una correlación canónica alta indica que las variables discriminantes permiten diferenciar entre los grupos, y en consecuencia, la función discriminará mucho, por lo cual se puede establecer que esta si discrimina de manera significativa, y viendo el análisis de este resultado de 0.650 nos muestra que sí está discriminando.

CUADRO 8

El autovalor y la correlación canónica

Eigervalues

\begin{tabular}{|l|r|r|r|r|}
\hline Function & Eigenvalue & \% of Variance & Cumulative \% & $\begin{array}{l}\text { Canonical } \\
\text { Correlation }\end{array}$ \\
\hline 1 &, $730^{\mathrm{a}}$ & 100,0 & 100,0 &, 650 \\
\hline
\end{tabular}

a. First 1 canonical discriminant functions were used in the analysis.

Seguimos ahora con el análisis de los grupos de centroides y coeficientes de las funciones, de acuerdo a esto para interpretar los signos de las ponderaciones resulta útil inspeccionar primero la ubicación de los centroides de cada grupo. Verificamos que el grupo de países con crecimiento tienden a obtener puntuaciones negativas en la función discriminante, mientras que los países sin crecimiento tienden a obtener puntuaciones positivas.

Sabiendo esto, la función discriminante nos indica que un incremento en el gasto público y en el índice de democracia (por encima de la media) hará más probable que el país obtenga una puntuación positiva y, con ello, que se ajuste al patrón de los países sin crecimiento. Por el contrario, un peso por debajo de la media será característico de un país con crecimiento. 
En cuanto a las variables índice de justicia y tasa de fertilidad, un valor por encima de la media hará disminuir la puntuación discriminante (dado que el signo es negativo) y será más característico de los países con crecimiento, y viceversa, una puntuación en estas variables por debajo de la media aumenta las posibilidades de que el país sea clasificado como un país sin crecimiento.

\section{CUADRO 9}

Centroides y coeficientes de función discriminante canónica estandarizada

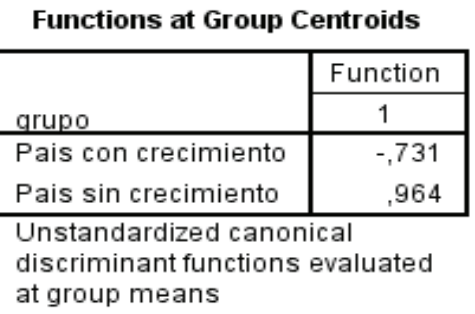

Standardized Canonical
Discriminant Function
Coefficients
\begin{tabular}{|l|c|}
\hline & Function \\
\cline { 2 - 2 } & 1 \\
\hline indice de justicia &,- 814 \\
gasto publico &, 559 \\
tasa de fertilidad &,- 607 \\
indice de democracia & 1,063 \\
\hline
\end{tabular}

En la siguiente tabla se muestran los coeficientes de la función discriminante canónica utilizados por el programa para calcular las puntuaciones discriminantes y la ubicación de los centroides en los grupos.

CUADRO 10

Coeficientes de función discriminante canónica no estandarizada

Canonical Discriminant Function
Coefficients
\begin{tabular}{|l|r|}
\hline \multicolumn{1}{|c|}{\begin{tabular}{|c|} 
Cunction \\
\cline { 2 - 2 }
\end{tabular}} & 1 \\
\hline indice de justicia &,- 046 \\
gasto publico &, 056 \\
tasa de fertilidad &,- 575 \\
indice de democracia & 8,837 \\
(Constant) & $-1,463$ \\
\hline
\end{tabular}

Unstandardized coefficients

Al solicitar los coeficientes de la función de clasificación se obtiene una función de clasificación para cada grupo. Para aplicar estos coeficientes se calcula cada una de las funciones para un sujeto dado y se clasifica al sujeto en el grupo en el que la función obtiene una puntuación mayor. 


\section{Ciro Calero / Marco Girio}

\section{CUADRO 11}

Siendo las funciones discriminantes lineales de Fischer:

Classification Function Coefficients

\begin{tabular}{|l|r|r|}
\hline \multirow{2}{*}{} & \multicolumn{2}{|c|}{ grupo } \\
\cline { 2 - 3 } & \multicolumn{1}{|c|}{$\begin{array}{c}\text { Pais con } \\
\text { crecimiento }\end{array}$} & $\begin{array}{c}\text { Pais sin } \\
\text { crecimiento }\end{array}$ \\
\hline indice de justicia &, 023 &,- 055 \\
gasto publico &, 203 &, 299 \\
tasa de fertilidad & 3,593 & 2,619 \\
indice de democracia & 22,855 & 37,833 \\
(Constant) & $-12,517$ & $-15,473$ \\
\hline
\end{tabular}

Fisher's linear discriminant functions

Función discriminante de países con crecimiento $=-12.517+0.023$ (índice de justicia) +0.203 (gasto público) +3.593 (tasa de fertilidad) +22.855 (índice de democracia)

Función discriminante de países sin crecimiento $=-15.473-0.055$ (índice de justicia) +0.299 (gasto público) +2.619 (tasa de fertilidad) +37.833 (índice de democracia)

Finalmente, se muestran los Resultados de la Clasificación. El porcentaje de casos correctamente clasificados es de 72,4\%. El 85\% de los países con crecimiento han sido clasificados correctamente, mientras que los países sin crecimiento han sido clasificados correctamente en un 55,6\%.

CUADRO 12

Classification Results ${ }^{a}$

\begin{tabular}{|c|c|c|c|c|c|}
\hline & & \multirow[b]{2}{*}{ grupo } & \multicolumn{2}{|c|}{ Predicted Group Membership } & \multirow[b]{2}{*}{ Total } \\
\hline & & & $\begin{array}{c}\text { Pais con } \\
\text { crecimiento }\end{array}$ & $\begin{array}{c}\text { Pais sin } \\
\text { crecimiento }\end{array}$ & \\
\hline \multirow[t]{4}{*}{ Original } & Count & Pais con crecimiento & 51 & 9 & 60 \\
\hline & & Pais sin crecimiento & 20 & 25 & 45 \\
\hline & $\%$ & Pais con crecimiento & 85,0 & 15,0 & 100,0 \\
\hline & & Pais sin crecimiento & 44,4 & 55,6 & 100,0 \\
\hline
\end{tabular}

a. $72.4 \%$ of original grouped cases correctly classified. 


\section{Conclusiones}

En el presente documento se analizan los determinantes de las tasas de crecimiento de los países de ingreso medio alto y medio bajo debido a la importancia de mucho de estos países como China, Brasil y otros países de interés como el Perú. Se utilizó la técnica de análisis discriminante encontrándose que los principales determinantes serían el gasto público, el índice de justicia, la tasa de fertilidad y el índice de democracia, reafirmando la vigencia del análisis realizado por Barro, documento base para el fundamento de nuestra investigación.

\section{Referencias bibliográficas}

Barro, Robert J. (1997) "Determinants of Economic Growth: A Cross - Country Empirical Study". Cambridge MA, MIT Press.

Barro, Robert J. (1999) "Determinants of Economic Growth: Implications of the global evidence for Chile". Banco Central de Chile.

Barro, Robert J. and Xavier Sala-i-Martin (1995) "Economic Growth". New York, McGraw Hill.

Moran, S. (2004) "Crecimiento económico: aspectos teóricos y evidencia empírica". Banco Central de Guatemala.

W.R. Dillon and M. Goldstein. "Multivariate Analysis: Methods and Applications". Wiley, New York, 1984. 